\title{
DESARROLLO HUMANO LOCAL EN NICARAGUA: EL CASO DE LA COOPERATIVA DE TURISMO RURAL COMUNITARIO “LAS PILAS-EL HOYO”: MUNICIPIO DE LEÓN
}

\author{
Carlos Santovenia Pérez*
}

Resumen: El objetivo de este trabajo fue evaluar en qué medida la Cooperativa de Turismo Rural Comunitario "Las Pilas-El Hoyo" como acción de aprovechamiento turístico contribuye al desarrollo humano local. Se basa en posiciones que reconocen la necesidad de recuperar la sabiduría colectiva y la inteligencia social, como bases del desarrollo endógeno, que el desarrollo se refiere a personas y por tanto tiene que ser a escala humana, de que una de las tareas más urgentes hoy es "restaurar un equilibrio adecuado entre la ciudad y la vida rural", y que "el lugar es la sede de la resistencia de la sociedad civil ante el deterioro". Dicha evaluación puede ser útil para mejorar la gestión de la cooperativa y las acciones en el territorio del Instituto Nicaragüense de Turismo (INTUR) y la Alcaldía. Se consultaron fuentes documentales y se entrevistóa actores claves en el proceso de creación y desarrollo de la cooperativa. La iniciativa ha contribuido a mejorar la vida de sus miembros, de la comunidad y a promocionar turísticamente el territorio; pero debe mejorar la preparación del personal, la incidencia local y el acceso al mercado. En conclusión: la cooperativa hace buen uso del potencial natural del territorio; debe fortalecer su estructura y funcionamiento y; el desarrollo del turismo rural comunitario es un proceso complejo y difícil en condiciones de atraso sociocultural y económico.

Palabras clave: Desarrollo humano; Turismo rural comunitario; Cooperativas de turismo; León, Nicaragua.

\section{DESENVOLVIMENTO HUMANO LOCAL EM NICARÁGUA: OCASO DA COOPERATIVA DE TURISMO RURAL COMUNTÁRIO "LAS PILAS-ELHOYO", MUNICÍPIO DE LEÓN}

Resumo: O objetivo deste trabalho foi avaliar até que ponto a Cooperativa de Turismo Rural Rural "Las Pilas-El Hoyo" como ação para o uso do turismo contribui para o desenvolvimento humano local. Baseia-se em posições que reconhecem; a necessidade de recuperar a sabedoria coletiva e a inteligência social, como bases do desenvolvimento endógeno, que o desenvolvimento se refere às pessoas e, portanto, tem que ser em escala humana, que uma das tarefas mais urgentes de hoje é "restaurar um equilibrio adequado entre a cidade e avida rural", eque" o lugar é o assento da resistência da sociedade civil diante da deterioração ". Esta avaliação pode ser útil para melhorar a gestão da cooperativa e ações no território do Instituto Nicaragüense de Turismo (INTUR) e da Prefeitura. Fontes documentárias foram consultadas e atores importantes foram entrevistados no processo de criação e desenvolvimento da cooperativa. A iniciativa contribuiu para melhorar a vida de seus membros, a comunidade e promover o turismo no território; mas deve melhorar a preparação do pessoal, incidência local e acesso ao mercado. Em conclusão: a cooperativa faz bom uso do potencial natural do território; deve fortalecer sua estrutura e funcionamento e; $\mathrm{O}$ desenvolvimento do turismo comunitário rural é um processo complexo e difícil em condições de atraso sócio-cultural e econômico.

Palavras-chave: Desenvolvimento Humano; Turismo rural comunitário; Cooperativas de turismo; León, Nicarágua.

\section{LOCAL HUMAN DEVELOPMENT IN NICARAGUA:THE CASE OF RURAL TOURISM-COMMUNITY COOPERATIVE "LAS PILAS-EL HOYO": MUNICIPALTYYOFLEÓN} of Rural Community Tourism "Las Pilas-El Hoyo" as an action for tourism use contributes to local human development. It is based on positions that recognize; the need to recover collective wisdom and social intelligence, as bases of endogenous development, that development refers to people and therefore has to be on a human scale, that one of the most urgent tasks today is "restore an adequate balance between the city and rural life, "and that" the place is the seat of the resistance of civil society in the face of deterioration". This evaluation can be useful to improve the management of the cooperative and actions in the territory of the Nicaraguan Tourism Institute (INTUR) and the Mayor's Office. Documentary sources were consulted and key actors were interviewed in the process of creation and development of the cooperative. The initiative has contributed to improve the lives of its members, the community and to promote tourism in the territory; but it must improve staff preparation, local incidence and market access. In conclusion: the cooperative makes good use of the natural potential of the territory; it must strengthen its structure and functioning and; The development of rural community tourism is a complex and difficult process in conditions of socio-cultural and economic backwardness.

Keywords: Human development; Community rural tourism; Cooperatives of tourism; León, Nicaragua.

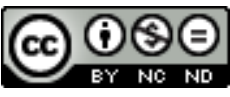

Licenciada por Creative Commons Atribuição Não Comercial / Sem Derivações/4.0/Internacional

* Experto Universitario en Gestión de la Calidad en MIPYMESTurísticas, Turismo (2010), por la Universidad de Universidad Carlos III Madrid/España. Máster en Gestión Pública del Turismo, Turismo (2000) por la Universidad Internacional de Andalucía/España. Licenciado en Educación, Geografía (1982) por la Universidad Pedagógica de Matanzas/Cuba. Profesor Titular. Departamento de Turismo. Facultad de Ciencias Económicas y Empresariales Universidad Nacional Autónoma de Nicaragua-León (UNAN-León). Campus Médico UNAN-León Telefax: (505)2311-0080 Ext. 18 Cel. 8885-8659 [carlosant@yahoo.com].

Rev. Latino-Am. Turismologia/RELAT, Juiz de Fora, v.3, n.1, pp.48-57, Jan./Jun. 2017 


\section{INTRODUCCIÓN}

Este trabajo es resultado del Curso: “(Re) Conocer el Desarrollo Local: Revisando paradigmas, Construyendo Desarrollo Humano Local en Nicaragua y América Central" ofrecido por la Universidad Complutense de Madrid y la UNAN-León.

Su objetivo fue evaluar en qué medida la Cooperativa de Turismo Rural Comunitario "Las Pilas-El Hoyo" como acción de aprovechamiento turístico contribuye al desarrollo humano local. Se basa en posiciones que reconocen la necesidad de recuperar la sabiduría colectiva y la inteligencia social, como bases del desarrollo endógeno, que el desarrollo se refiere a personas y por tanto tiene que ser a escala humana, de que una de las tareas más urgentes hoy es "restaurar un equilibrio adecuado entre la ciudad y la vida rural", y que "el lugar es la sede de la resistencia de la sociedad civil ante el deterioro".

El estudio documental y la información obtenida de actores claves del proceso de creación y establecimiento de la Cooperativa "Las Pilas-El Hoyo", indican que esta; creada como resultado de las acciones emprendidas en el Municipio de León como respuesta a los efectos del Huracán Micht; es una experiencia positiva en la mejora de la calidad de vida de un grupo de campesinos y de cómo iniciativas locales pueden impulsar sectores económicos como el turismo.

La cooperativa enfrenta dificultades; relaciones institucionales, estancamiento en áreas de su quehacer, falta de estrategias más efectivas, dinámica social marcada por la "cultura de duelo". Sin embargo, su presencia ha hecho de la zona uno de los sitios más visitados de Occidente, y ha mejorado las condiciones del ambiente. La aplicación de estrategias adecuadas puede elevar la eficacia de la cooperativa como experiencia de desarrollo local enfocado a las personas.

\section{MARCOTEÓRICO}

\subsection{Desarrollo y Desarrollo Humano}

Al consultar la literatura sobre desarrollo humano nos encontramos con que para hablar de este tipo de desarrollo se debe partir de la definición general de desarrollo; definición que es considerada algo inasible por algunos especialistas en la materia. Esto debido a que, como expresa Francisco González Cruz en su ensayo, Desarrollo humano sustentable local; "El término "desarrollo" ha recibido numerosos calificativos cuando se refiere a la persona humana, a la sociedad, a las naciones o a las comunidades: desarrollo humano, armónico, integral, sustentable o sostenible, endógeno y muchos otros". Y agrega que; "[...] se han ensayado numerosas definiciones, desde meramente cuantitativas hasta únicamente cualitativas."

Eduardo García Docampo, en Perspectivas teóricas en Desarrollo Local, expone que las bases de la moderna teoría del desarrollo económico se sentaron "Tras una larga etapa de formación de teorías del crecimiento económico, que se iniciaría con los trabajos pioneros de Adam Smith, John Stuart Mill, Malthus o Marx, por citar sólo algunos ejemplos, [...]". Estas teorías, en su mayoría, contienen la idea de que el desarrollo "lleva aparejado crecimiento económico", e incluso llegan a considerar al crecimiento económico y al desarrollo como sinónimos. Situación que se mantiene hasta hoy, y que es una de las principales causas de la intensa discusión sobre que es desarrollo. El cual sigue siendo concebido en muchos casos a partir del crecimiento de la renta per cápita.

Una explicación plausible del sostenido y amplio debate sobre el desarrollo económico es la idea planteada por Antonio Vázquez Barquero "La conceptualización del desarrollo económico evoluciona y se transforma a medida que lo hace la sociedad, a medida que los países, regiones y ciudades tienen que dar solución a nuevos problemas, a medida que las innovaciones y el conocimiento se difunden por las organizaciones económicas y sociales." (Vázquez Barquero, 2007)

En este trabajo no se pretende ni es posible abordar esa discusión, que cuenta con una amplia literatura. Sino que se trata de dar una definición de desarrollo humano lo más aceptable posible, así como una de desarrollo local que ayude a sustentar la investigación prevista.

En el trabajo antes mencionado de Francisco González Cruz, este plantea que un concepto muy apropiado de desarrollo humano; "se encuentra en el siempre actual libro "El Desarrollo a Escala Humana" escrito por Manfred Max-Neef, Antonio Elizalde y Martín Hopenhayn (1980): "Tal desarrollo [el desarrollo a escala humana] se concentra y sustenta en la satisfacción de las necesidades humanas fundamentales, en la generación de niveles crecientes de autodependencia y en la articulación orgánica de los seres humanos con la naturaleza y la tecnología, de los procesos globales con los comportamientos locales, de lo personal con lo social, de la planificación con la autonomía y de la Sociedad Civil con el Estado". 
Sin embargo, pareciera que el mismo no está satisfecho totalmente con esa definición y reconoce; "que desde esa fecha muchos expertos han aportado valiosas ideas," y destaca como el más novedoso de ellos a Amartya Sen con su concepción de que: "[...] el desarrollo puede concebirse [...] como un proceso de expansión de las libertades reales de las que disfrutan los individuos" (Sen, 2000)." Idea que retoma y precisa el Programa de las Naciones Unidas para el Desarrollo; "el desarrollo humano es un proceso a través del que los ciudadanos amplían sus capacidades de tal forma que permita alargar su vida, mejore sus conocimientos y tenga los recursos económicos necesarios para llevar una vida digna." (Vázquez Barquero, 2007)

A continuación, se presenta un párrafo algo extenso de González Cruz, donde sentencia que:

"La palabra desarrollo adquiere realmente su sentido en la medida que se relaciona con la persona humana, porque el destinatario único y determinante de todo desarrollo, para que se precie de tal, es y debe ser la persona humana. No puede existir un desarrollo económico si no hay desarrollo humano. No hay desarrollo sustentable, endógeno, sostenible, integral o como se le quiera adjetivar, si no está dirigido a la defensa de la dignidad de la persona humana. Y esto es muy importante tener siempre presente, por cuanto con demasiada frecuencia se instrumentan políticas que soslayan a la persona humana en aras de la prosperidad económica."

La extensa cita anterior se justifica a partir de que, en el caso del turismo hay muchos ejemplos de, "desarrollos sostenibles, integrales, etc.", en los que la dignidad humana ha sido relegada y olvidada en aras de una falsa "prosperidad económica".

\subsection{Desarrollo Local}

La teoría del desarrollo local es otro de los grandes campos teóricos en las ciencias sociales actuales. Diferentes autores destacan que la discusión del desarrollo ha ido evolucionando, y que en las últimas décadas la atención se ha ido centrando en el desarrollo local, a partir del auge de las posiciones que defienden el desarrollo como un proceso endógeno.

Vázquez Barquero, en su trabajo para la CEPAL del año 2000, retoma su propia definición de desarrollo local; "Se puede definir el desarrollo económico local como un proceso de crecimiento y cambio estructural que mediante la utilización del potencial de desarrollo existente en el territorio conduce a la mejora del bienestar de la población de una localidad o una región. Cuando la comunidad local es capaz de liderar el proceso de cambio estructural, la forma de desarrollo se puede convenir en denominarla desarrollo local endógeno. (Vázquez Barquero, 1988).

Esta atención al desarrollo local está marcada por las nuevas condiciones para la actuación económica social creadas por la globalización, como expresa Francisco González Cruz en "Desarrollo humano sustentable local" (2009), de las que numeramos las principales:

1. "Si ahora la gente tiene posibilidades de acceso desde su lugar, este tiene un nuevo valor. Ya la gente no está estructuralmente bloqueada si no vive en un lugar que no sean aquellos pocos privilegiados por los procesos de concentración previos a la revolución tecnológica. Ahora puede relacionarse desde cualquier parte."

2. "Entonces la calidad de la vida local tiene una nueva e inusitada dimensión. La gente tiene derecho no solo a una aceptable calidad de vida local, sino que esta le dé la posibilidad de acceso a lo global. Ya no cuenta que la localidad le ofrezca solamente aceptables servicios públicos y alguna que otra ventaja. Ahora cuenta mucho que la localidad garantice a la gente, además, posibilidades reales de competitividad a escala planetaria."

3. "Las consideraciones relativas a la "lugarización" tiene varias consecuencias, tanto en el orden social, político-administrativo, económico y en otros órdenes. La gente se convence que su calidad de vida y su prosperidad dependen fundamentalmente de su propio esfuerzo y entonces se organiza para hacer las cosas que considera necesario. No espera que las autoridades resuelvan los problemas."

Dichas condiciones son de gran relevancia en el caso de la actividad turística, que es hoy una de las más significativas en la economía mundial. Todavía, otras consideraciones importantes sobre el desarrollo local son las planteadas por Manuel García Docampo (2007), en "Perspectivas teóricas en Desarrollo Local". Muchas de estas pueden utilizarse como guías del accionar en un proceso de desarrollo local.

Este autor plantea que; "Una iniciativa de desarrollo económico local no es únicamente un proyecto exitoso en un territorio. Se requiere una concertación institucionalizada de los actores públicos y privados locales más relevantes, compartiendo una estrategia de desarrollo común. Por otra parte, una 
suma compleja de instrumentos de fomento no puede sustituir nunca la necesaria institucionalidad territorial para el desarrollo económico. No es lo mismo crear instrumentos desde el nivel central, los cuales pueden ser utilizados por los diferentes territorios, que impulsar y promover un protagonismo y capacidad mayores de dichos territorios para un mejor aprovechamiento de sus recursos endógenos."

Estas relaciones público-privadas son algo esencial en el turismo, donde por lo general el sector privado hace aprovechamiento de recursos culturales o naturales que son propiedad pública o al menos comunitaria, ya que la mayoría de las empresas del sector son de carácter privado.

Otra línea de acción indicada por García Docampo es la; "Conformación de un núcleo inicial de personas que comparten una situación (necesidades, carencias, valoraciones, etc.) que desean superar. Tiene que haber una predisposición inicial que vincule a unas personas o grupos ante algo que no les convence y que abra caminos de convergencia entre los más decididos. La participación debe generarse desde el principio, aunque sea con poca gente, con un buen ambiente construir confianzas para después ampliarla. Es preferible ir cargando las baterías en el proceso, que no empezar con mucha gente y con mucha energía y que esta se vaya descargando por mal ambiente."

En este mismo tenor orientativo, García Docampo señala que el desarrollo local desde las comunidades debe incluir, la "Realización de un plan de trabajo con un grupo motor. El grupo motor es un grupo mixto de las personas voluntarias y de los profesionales que se van a poner a trabajar".

El mencionado autor hace una precisión en cuanto a las políticas de desarrollo local; "Las políticas de desarrollo local no se limitan únicamente al desarrollo municipal. Igualmente, aunque puede parecer una cuestión obvia, el desarrollo municipal se refiere no sólo al núcleo urbano del municipio, sino a la totalidad de la población diseminada en todo el territorio municipal. Esta es una cuestión fundamental cuando se trata de atender, sobre todo las demandas de la población dispersa en comunidades campesinas, muchas veces ignorada o marginada de los procesos de desarrollo." Algo también muy importante en procesos de desarrollo local basado en el turismo, pues para muchos el turismo sigue siendo una actividad fundamentalmente urbana, sobre todo al tomar en cuenta que la mayoría de los centros de recepción y distribución de visitantes están ubicados en sitios urbanos, aunque los atractivos que generan la demanda estén en el área rural.

Por último, Docampo llama la atención de la importancia del papel que corresponde a las instituciones educativas en el impulso del progreso local, cuando expresa: "Con todo, hay que superar cuanto antes el desencuentro existente entre la oferta de capacitación de universidades y centros de formación y las necesidades de innovación en los diferentes sistemas productivos locales.

Para ello, es fundamental la incorporación activa de las entidades educativas, universitarias y de investigación científica y tecnológica en las iniciativas de desarrollo económico local. La actuación decidida de los actores territoriales es la única capaz de construir nexos entre la oferta de conocimiento y los usuarios últimos o demandantes en los diferentes sistemas productivos locales." Situación que también es de gran incidencia en el turismo, sobre todo en las zonas rurales, donde al lamentablemente extendido bajo nivel educativo, se une un desconocimiento casi total de las actividades relacionadas con la presencia de visitantes.

\section{METODOLOGÍA}

Para obtener la información se consultaron fuentes documentales, tomadas en su mayoría de Internet, sobre todo para caracterizar el territorio (A). Se entrevistó a actores clave del proceso de creación y desarrollo de la Cooperativa de Turismo Rural Comunitario Las Pilas-El Hoyo; la coordinadora de Ecología y Desarrollo, la presidenta de la cooperativa, tres miembros activos de la cooperativa y la directora del Departamento de Turismo de la Alcaldía Municipal de León. Además, se aplicó un instrumento elaborado en el curso, cuya estructura guía la presentación de los resultados, y que se presenta resumido a continuación.

\subsection{Instrumento aplicado}

\section{B. Abordajes:}

B.1. Modo: Modo o modelo de desarrollo (Comunitario, público, privado) Procesos: Como se hizo, procesos actuales.

B.2. Actores: nacionales, locales, regionales, internacionales, instituciones públicas y privadas, profesores, investigadores, mujeres, ancianos, jóvenes. 
B.3. Territorio: Estructura, organización, funcionamiento (en lo natural y en lo económico y cultural). La gente en el territorio.

B.4. Estrategias: estrategias aplicadas. Su funcionamiento. Claves

B.5. Acciones/Instrumentos: (Como hacen el trabajo del día a día)

B.6. "El vivir"/Cotidiano: La vida cotidiana; problemas, alegrías, obstáculos, luchas, triunfos, los compartir.

B.7. Lecciones aprendidas: Referencias para otras experiencias.

B.8. Aportaciones científicas: qué hace falta investigar, que problemas científicos se identifican, análisis territoriales que se pueden hacer (naturales o geoeconómicos), aportes teóricos o metodológicos

\section{B.9. Buenas Prácticas de Desarrollo Humano Sostenible}

C. Plan. (Establecer un plan de trabajo para mejorar la experiencia)

Este estudio podría ser mejorado en aspectos como: impacto del accionar de la cooperativa en las comunidades aledañas, relaciones con instituciones públicas y, la incidencia sobre la vida de los habitantes del territorio, de los recursos invertidos por la cooperación internacional en el turismo.

\section{RESULTADOS}

\section{1-Modo o modelo: Procesos}

El Parque Nacional Complejo Volcánico Pilas - El Hoyo (11,532.2 hectáreas), se encuentra ubicado en la Región del Pacífico de Nicaragua, en el Departamento de León, con las coordenadas geográficas (WGS 84):

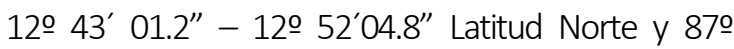
24'03.6" - 870 34'04.8" de Longitud Oeste. La Cooperativa administra la infraestructura construida al oeste de la base del complejo volcánico.

La creación de la cooperativa deriva de las acciones tomadas después del Huracán Mitch (Octubre, 1998). Al Hermanamiento de Zaragoza y ECODES (Fundación Ecología y Desarrollo) les fue asignado el territorio de León Noreste (14 comunidades rurales). Para su accionar en la mitigación del desastre, ECODES creó el Proceso de Desarrollo Integral (PDI) en 2001, que abarcaba diferentes componentes socioeconómicos. Durante la identificación de potenciales endógenos, se determinó que; el área protegida era un recurso con un gran potencial que podría ser desarrollado y repercutir positivamente en la economía campesina. Primero se identificó el potencial agroecológico, lo turístico aparece después.

En 2001-2002 se madura la idea; se hace un estudio más profundo del territorio, se reconocen los recursos turísticos, posibles rutas y las potenciales relaciones agricultura-turismo en torno a una zona volcánica. Se inicia un proceso de formación de los implicados con la Escuela de Turismo de la UNAN-León, y viajes de evaluación de las rutas definidas. La comunidad impulsó la formulación y ejecución de 2 proyectos, uno de infraestructura y otro de promoción del turismo rural comunitario en la reserva; subvencionados por el Gobierno de Aragón en sus convocatorias 2003 y 2004. Y los interesados en el proyecto se organizaron en una cooperativa, Proceso apoyado por ECODES y la Alcaldía Municipal de León. El estatus legal de la cooperativa en la reserva, es un permiso de administración de la infraestructura concedido por la Alcaldía.

Basados en las condiciones de desarrollo del turismo, se creó una cooperativa de turismo rural comunitario, que, según los actores, se funda en los siguientes principios:

1. Solidaridad, que permite una distribución local y equitativa de los recursos generados;

2. Conciencia ambiental, que promueve un uso adecuado de los recursos naturales;

3. Trabajo comunitario organizado, que estrecha y refuerza los vínculos ínter-comunitarios fortaleciendo así las redes locales;

4. Recuperación de la identidad local, que realza las costumbres, las tradiciones y la cultura de la comunidad receptora.

Para ambos proyectos, se consideró como beneficiarios directos a la población que habita en la zona rural noreste, aproximadamente 5,075 personas, en ese entonces. $Y$ como beneficiarios indirectos; el sector servicios relacionado con el turismo. Estos proyectos se ejecutaron en el período de marzo 2004 a febrero 2007; con un presupuesto total de 175.911,50 € (Gobierno de Aragón 138.231,50€; resto de socios $37.680,00 €)$. La contraparte local fueron la Alcaldía Municipal de León y la Cooperativa (ECODES, 2014). 
En 2004-2005 se construyó el Centro de Interpretación de los Maribios(CIM); centro de información e interpretación de la cordillera, desde el que se realiza trabajo de protección de la reserva natural. Este usa energía solar.

La presencia del CIM y la cooperativa ha generado un crecimiento significativo de las visitas a la reserva; 13, 801 personas en 2013 (Libro de visitantes del CIM), que llegan principalmente para ascender al Cerro Negro y realizar sandboarding, (deslizarse con una tabla similar a la de snowboard por la ladera del volcán). Esto ha impulsado los estudios del territorio y de la cooperativa; realizados por instituciones especializadas y las universidades con carreras de turismo. La iniciativa de turismo rural generó interés entre los jóvenes de la comunidad; varios se han graduado en turismo, aunque al momento del estudio ninguno trabajaba de forma permanente con la cooperativa.

La Cooperativa es fundadora de la Red Nicaragüense de Turismo Rural Comunitario (RENITURAL).

\subsection{Principales Actores en el Territorio}

1) Internacionales: ECODES, Hermanamiento de Zaragoza, Gobierno de Aragón.

2) Comunidades del territorio y sus líderes;

3) CISAS (Centro de Información y Servicios de Asesoría en Salud).

4) Cooperativa del Campo (agricultura orgánica).

5) Cazadores furtivos: comunitarios y personas de los municipios aledaños. Cazan mamíferos y/o aves, y generan incendios.

6) Cortadores de leña: de comunidades contiguas a la reserva, talan ilegalmente.

7) Policía Nacional: La cooperativa alega tener poco apoyo de la policía en el combate a la caza y tala ilegales.

8) MARENA (Ministerio del Ambiente y los Recursos Naturales): responsable de las áreas protegidas. Sus relaciones con la cooperativa no son buenas. $\mathrm{Ha}$ dado los permisos para establecer las infraestructuras existentes.

9) Alcaldía Municipal de León: El proyecto es resultado de sus acciones en el área tras el Huracán Micht. Después de algunas tensiones, las relaciones entre ambas instituciones son buenas, la Alcaldía gestiona apoyos económicos a la cooperativa.
10) INTUR: mantiene estrechas relaciones con la cooperativa. Facilita capacitaciones y apoyo en la promoción turística.

11) UNAN-León: Capacitación a los cooperativistas y otras iniciativas. Ejecuta estudios sobre la cooperativa y el desarrollo del turismo en el territorio.

12) Ministerio de Salud: Su presencia es débil, la mayor parte del área es atendida a través de brigadas médicas con visitas mensuales.

13) Ministerio Agropecuario y Forestal: Presente en algunas zonas del área protegida con el Plan Libra por Libra.

14) Instituto Nicaragüense de Estudios Territoriales (INETER): Monitorea la actividad de los volcanes durante las 24 horas.

15) Municipalidades de La Paz Centro y Malpaisillo: Son alcaldías pequeñas; tienen menor capacidad de gestión que la de León.

\subsection{Territorio}

\subsubsection{Estructura y funcionamiento natural}

La categoría de manejo del área natural (Plan de Manejo, MARENA, 2008)) es la de "Parque Nacional", que técnica y legalmente se sustenta en 3 especies de fauna como objeto de conservación; el Tigrillo (Leopardus tigrina), la lora nuca amarilla (Amazona auropalliata) y el mono cara blanca (Cebus capucinus), 2 especies de flora en peligro de extinción; la Caoba del Pacífico (Swietenia humilis) y la tuna (Opuntia decumbens), 11 tipos de ecosistemas, los volcanes activos, un volcán antiguo (El Hoyo), una laguna cratérica, numerosos paisajes de belleza excepcional y la nula presencia de asentamientos humanos dentro del área. Las principales afectaciones al ambiente son: uso inadecuado del suelo, agricultura intensiva, extracción de leña, cacería, extracción de fauna, desarrollo de actividades turísticas sin control.

\subsubsection{Estructura y funcionamiento socioeconómico}

Administrativamente el Parque Nacional está ubicado en los municipios de León, La Paz Centro y Larreynaga. Los principales núcleos de población se concentran en la Zona de Amortiguamiento, con aproximadamente 4,568 personas (1,002 familias). Pertenece a León el $63 \%$ de la población, a Malpaisillo el $24 \%$ y Telica y La Paz Centro, el $7 \%$ y $6 \%$, respectivamente. El $19.14 \%$ son menores de 10 años y un $80.86 \%$ constituyen la Población en Edad de 
Trabajar (10 años y más). De la población en edad de trabajar, un 52.24\%, está en condición de inactividad; amas de casa, estudiantes a tiempo completo o jubilados y retirados. El desempleo imperante en la zona queda cubierto bajo el manto del sub empleo; en la población más adulta en trabajos "por cuenta propia" y en los más jóvenes en trabajos "sin pago" para garantizarse su alimentación. Las principales actividades económicas son las agropecuarias (41\%) y la extracción de leña (24.7\%) (MARENA, 2008).

Hay serios problemas con el agua para uso humano en la mayor parte del área: escasez de fuentes de abastecimiento y mala calidad del agua. Todas las comunidades tienen caminos de penetración que las comunican con sus cabeceras municipales y la cabecera departamental. El servicio de energía eléctrica se presta en el $66 \%$ de las comunidades. El $8 \%$ de los entrevistados no disponen de ningún tipo de alumbrado durante la noche (Plan de Manejo, 2008).

En toda la zona de amortiguamiento sólo existe una Unidad de Salud en la comunidad Miramar. Casi todas las comunidades poseen infraestructura escolar con servicio de primaria incompleta, hasta 4to grado de primaria, y en otras la primaria completa. Los centros de enseñanza secundaria están fuera del área de estudio. La tenencia de la tierra se encuentra altamente concentrada en manos de grandes productores con áreas mayores de 500 manzanas.

\subsection{Estrategias Aplicadas}

1) Capacitación en temas sobre turismo y gestión de empresas;

2) Incorporación a la RENITURAL y otras organizaciones de turismo locales;

3) Que los guías sean siempre de las comunidades;

4) Basarse en el análisis interno de la población sobre su potencial;

5) Fortalecimiento de las decisiones y capacidades para su propio crecimiento;

6) Dinamización e incorporación de los actores relacionados al turismo, aunque hubo dificultades (con la Alcaldía y el MARENA). (Estrategia CLAVE).

\subsection{Acciones/Instrumentos}

El trabajo del día abarca cuidar el CIM Los Maribios, limpiar el centro, atender a los clientes, darle mantenimiento a las tablas de sandboarding, alimentar a las iguanas, sembrar en el área del albergue. Estas son la base del funcionamiento de la cooperativa.

\section{6 "El vivir"/Cotidiano}

Entre las alegrías alcanzadas están: tener un trabajo permanente por 14 años, poder comprar un camión (usado), un tractor para sembrar al borde de la reserva, tienen 4 trabajadores de las comarcas en el albergue, el 3 de junio (2014) ofrecieron diversas actividades a un grupo de niños del Valle de los Caleros: piñata, paseo por el Cerro Negro.

Obstáculos principales: las relaciones con el MARENA, que generan conflictos con los demás comunitarios y limita las acciones coordinadas de protección de la reserva, y da pie a las touroperadoras para tratar de restar beneficios a la cooperativa. Falta apoyo de la alcaldía para reparar los caminos. Las vacas suben a pastary dañan la reforestación. Ninguno de los miembros de la cooperativa habla inglés. El albergue está estancado por problemas administrativos. Tienen problemas con el mercadeo; no venden paquetes, sólo atienden a las touroperadoras que llegan con sus turistas.

Los cooperativistas incluyen entre sus triunfos: 90\% de reducción de los incendios en el área, trabajando en coordinación con Defensa Civil, el Ejército y las comunidades. Como logros especiales están, la llegada de más turistas; 2008: 350-400 personas por semana, 2014: 700-800 personas en la semana. Y la exposición que esperan montar con material fotográfico de cómo estaba el área hace 14 años y los logros en la protección del medio ambiente.

\subsection{Lecciones aprendidas (Referencias para otras experiencias)}

1) Un grupo de campesinos, con la capacitación adecuada puede llevar adelante un proyecto turístico con éxito relativo.

2) La protección que se ha dado al área y a los animales; "la gente viene interesada en conocer la naturaleza...".

3) El tema de la basura. Los extranjeros, están conscientes de cómo manejar la basura; pero la mayoría de los nacionales no.

4) Han aprendido a no quemar la basura, sino enviarla directo al basurero.

5) Es necesario sanar los territorios para poder ofrecer un mejor desarrollo local. 
6) Muy difícil crear y desarrollar iniciativas como esta en el contexto de esa época.

7) El ritmo del proceso hasta llegar a vender turismo fue rápido, desde la cooperativa, comparado con el ritmo de las instituciones.

8) Las instituciones demoraron en comprender como la iniciativa podría contribuir a aprovechar el turismo de forma sostenible y generar desarrollo local.

\subsection{Aportaciones científicas}

\subsubsection{Qué hace falta investigar}

El efecto de la "cultura multiduelo" (Visión de la vida como resultado de la acción sistémica e intensiva de fenómenos y/o hechos destructivos como huracanes, guerras, terremotos); como generadora de obstáculos al desarrollo humano local.

Otro tema importante es el impacto económicosocial de las inversiones en turismo en el área $(\approx$ 200.000.00€)

\subsubsection{Problemas científicos que se identifican}

La relación salud social-Desarrollo Humano Local parece ser el principal Problemas científicos que se identifican. Además, también se puede hacer un análisis territorial que se pueden hacer (naturales o geoeconómicos). En lo particular, a lo elemento natural, se debe enfocar la atención en la implementación del turismo en áreas naturales protegidas, cercanas a núcleos poblados y sin protección puede ser una manera adecuada de generar el cuidado y mejoramiento ambiental de estas.

\subsubsection{Aportes teóricos o metodológicos}

Otro tema interesante es el efectivo proceso de desarrollo humano local que requiere como condición básica el saneamiento social del territorio.

\subsection{Buenas Prácticas de Desarrollo Humano Sostenible (DHL)}

El sistema de captura de agua de lluvia para abastecer el CIM, creación de zoocriaderos, apoyo a niños de la zona con útiles escolares, relaciones con otros comunitarios (generación de oportunidades de empleo y compras de productos y servicios)

\subsection{Plan. (Propuesta de acciones para mejorar la experiencia)}

1) Incrementar la participación de las comunidades en la actividad turística alrededor del Complejo Volcánico.

2) Mejorar la estructura y funcionamiento de la cooperativa incorporando jóvenes de la zona con formación.

3) Crear un COMANEJO que dé más seguridad y claridad a la cooperativa sobre su papel en el área.

4) Contratar un especialista en mercadeo.

5) Capacitar a los comunitarios para ofrecer servicios a los turistas.

6) Capacitar en idioma inglés a los miembros de la cooperativa.

7) Mejorar los mecanismos de toma de decisiones y las capacidades de negociación y resolución de conflictos de la cooperativa.

8) Estudios de flora y fauna de la zona para mejorar el conocimiento de la misma

9) Capacitar al personal de la cooperativa en los nombres científicos de las especies

10) Incrementar el impacto de los fondos de la cooperación invertidos en la zona con una mayor incidencia del proyecto en la vida de las comunidades.

\subsection{Discusión de los Resultados}

El desarrollo de este proyecto, que inicia como resultado de las medidas implementadas para paliar las consecuencias de un desastre; pone de relieve las significaciones de la lugarización; en primer lugar, porque el grupo de campesinos involucrados en la cooperativa comprende que a pesar de cierta dureza del territorio donde viven, pueden luchar por crear mejores condiciones. Y reclaman a las autoridades, pero no cejan en su lucha. Y paradójicamente, las "desfavorables" condiciones naturales del territorio constituyen específicamente el potencial que les permite lograr posiciones competitivas a nivel planetario como destino turístico de naturaleza y aventura. Posición que también está sustentada en que los aspectos tecnológicos les permiten darse a conocer a nivel internacional por medio de Internet.

En el caso de la Cooperativa Las Pilas-El Hoyo, estamos en presencia de un proyecto exitoso en un territorio, en este caso de turismo rural comunitario en Nicaragua, como plantea García Docampo, pero 
debemos agregar que su éxito se debe precisamente a la "concertación institucionalizada de los actores públicos y privados locales más relevantes, compartiendo una estrategia de desarrollo común"; la Alcaldía Municipal de León, la ONG Ecología y Desarrollo, el Hermanamiento de Zaragoza-León, las comunidades de León Noreste, el INTUR, entre otras. llustra esto la concesión del permiso de administración de la reserva natural a la cooperativa, por parte de la Alcaldía.

Otra de las propuestas de Docampo; la conformación de un núcleo inicial de personas que comparten una situación, que desean superar, en el caso abordado funcionó al revés; pues la cooperativa inició con más de veinte personas y al momento del estudio contaba con once miembros, ya que algunos desacuerdos y las duras condiciones de trabajo iniciales provocaron que muchos abandonaran el proyecto. Aunque los que quedaron no perdieron la energía y continúan trabajando. La creación de este grupo requiere, como se puso de manifiesto en el trabajo, que previamente se busque mejorar la salud social del territorio; sobre todo en cuanto a organización y funcionamiento de la localidad, para reducir los conflictos que pueden entorpecer el desarrollo endógeno.

La lección aprendida de que un grupo de campesinos, con la capacitación adecuada puede llevar adelante un proyecto turístico con éxito relativo; pone de manifiesto el papel de las universidades y las instituciones de formación en las iniciativas de desarrollo económico local. Los miembros de la cooperativa recibieron capacitaciones del Departamento de Turismo de la UNAN-León; en una experiencia muy enriquecedora para sus docentes, que tuvieron que adaptar los contenidos y técnicas didácticas, a un grupo de personas que en muchos casos no sabían leer y escribir; experiencia que pudieron mejorar y aplicar exitosamente en otras iniciativas de desarrollo turístico rural.

El hecho de que "el desarrollo municipal se refiere no sólo al núcleo urbano del municipio, sino a la totalidad de la población diseminada en todo el territorio municipal"; es la base del impulso recibido por este proyecto de parte de las instituciones municipales y nacionales implicadas, sin embargo, es necesario destacar que dicho apoyo ha sido insuficiente, pues las tour operadoras que llevan visitantes al Centro de Interpretación, son las principales beneficiadas por el turismo que se desarrolla en la reserva natural, mientras los esfuerzos en su conservación recae en los comunitarios.

Al confrontar lo expuesto por Vázquez Barquero, acerca del desarrollo local con las condiciones que ha ido logrando el área rural donde se desempeña la cooperativas Las Pilas-El Hoyo, encontramos que el crecimiento de la actividad turística ha sido sustancial; de ninguna visita a miles de visitas al año, el cambio estructural se manifiesta en las instalaciones turísticas que han pasado de cero a dos, han mejorado los caminos, y de una zona de agricultura deprimida ha evolucionado a uno de los destinos de turismo de naturaleza y aventura más importantes del país. Todo esto, basado en el potencial que para ambos tipos de turismo posee la reserva natural. Sin embargo, la mejora del bienestar de la población de la localidad puede superarse, esto es una debilidad del proyecto; $y$ la vía principal para incrementar el buen vivir de los comunitarios aledaños al área es a través de una mayor participación directa de estos en las actividades turísticas y como suministradores de servicios y productos a la cooperativa, a los turistas y a las empresas que traen turistas a la reserva natural.

Como resumen del análisis podemos emplear la definición de desarrollo humano del Programa de las Naciones Unidas para el Desarrollo; "el desarrollo humano es un proceso a través del que los ciudadanos amplían sus capacidades de tal forma que permita alargar su vida, mejore sus conocimientos y tenga los recursos económicos necesarios para llevar una vida digna." Los miembros de la cooperativa y algunos habitantes de las comunidades aledañas, estos últimos menos de los deseados; han podido ampliar sus capacidades; hoy son capaces de realizar diferentes funciones administrativas, empresariales, de gestión del territorio, han aprendido sobre turismo y otras actividades como la conservación ambiental, sobre la historia del área que aprovechan y han adquirido recursos monetarios, recursos materiales como equipos de transporte, mejores viviendas, mejor acceso a la salud, entre otras condiciones que han ido dignificando sus vidas.

\section{CONCLUSIONESY/O RECOMENDACIONES}

Teniendo en cuenta que el objetivo de este trabajo fue evaluar como la Cooperativa de Turismo Rural Comunitario "Las Pilas-El Hoyo", contribuye al desarrollo humano local, se ha podido verificar que: 
- El uso agroecoturístico del territorio es un acierto, ya que está en consonancia con lo expresado en los mapas de uso potencial del suelo del área.

- La cooperativa debe aprovechar a los jóvenes del territorio que cuentan con formación, especialmente en turismo, para fortalecer las estructuras y funcionamiento de la misma.

- El desarrollo del turismo rural comunitario es un proceso difícil en condiciones de atraso sociocultural y económico, que requiere de la creación previa de condiciones sociales y de formación técnica básica.

- La cooperativa debe seguir trabajando en profundizar el cumplimiento de los principios del turismo rural comunitario, para consolidar su contribución al desarrollo local humano.

\section{REFERENCIAS}

Carpio Martín, José (2000). Desarrollo local para un nuevo desarrollo rural. Anales de Geografía de la Universidad Complutense, 20: 85-100.

Entrevista a Emir Munguía y Fabio Manuel Membreño Leytón (8/06/2014). Integrantes de la Cooperativa de Turismo Rural Comunitario Las Pilas-El Hoyo. Centro de Interpretación Los Maribios.

Entrevista a Flor Danelia Velásquez (2/06/2014). Presidenta de la Cooperativa de Turismo Rural Comunitario Las Pilas-El Hoyo. Oficina de la Cooperativa en León.

Entrevista a María de Jesús Delgado (2/06/2014). Directora del Departamento de Turismo. Alcaldía Municipal de León. Oficina del Departamento.

Entrevista a Olga Ara del Amo (21/05/2014). ECODESOficinas ECODES, León, Nic.

ECODES. Fortalecimiento del Turismo Rural Comunitario en la Reserva Natural Las Pilas-El Hoyo. (León-Nicaragua) http://www.ecodes.org/proyectos-

finalizados/fortalecimiento-del-turismo-rural- comunitario-en-la-reserva-natural-las-pilas-el-hoyoleon-nicaragua\#.U2Qsg1V5OW5. Consultada el 2/05/2014

García Docampo, Manuel (2007). (Editor) Perspectivas teóricas en Desarrollo Local. Netbiblo, S. L. (La Coruña) España

González Cruz, Francisco (2012). Desarrollo humano sustentable local», Polis [En línea], 22 | 2009, Publicado el 08 abril 2012, consultado el 30 septiembre 2016. URL: http://polis.revues.org/2598

Laboratorio de Microbiología de Agua (2014?). Facultad de Ciencias. UNAN-León. Informe del diagnóstico preliminar de la calidad del agua de consumo en las comunidades del sector rural noreste del municipio de León.

http://www.cisas.org.ni/files/Informe\%20Final\%20EC ODES-

UNAN\%20Agua\%20Sector\%20Rural\%20NE\%20Leon. pdf.Consultado en 2/06/2014

MARENA - Ministerio del Ambiente y los Recursos Naturales. (Agosto 2008). Plan de manejo Complejo Volcánico Pilas-El Hoyo http://www.sinia.net.ni/wsinap/documentos/PM/Pla n_de_Manejo_Pilas_El_Hoyo.p df Consultado 10/06/2014.

MARENA - Ministerio del Ambiente y los Recursos Naturales. (Agosto 2008). Reserva Natural Complejo Volcánico Las Pilas-El Hoyo. http://www.marena.gob.ni/pacificonorte-region-pacifico-523/rn-complejo-el-hoyoregion-pacifico-542. Consultada el 2/05/2014

Vázquez Barquero, Antonio. (2000). Desarrollo económico local y descentralización: aproximación a un marco conceptual. Proyecto CEPAL/GTZ "Desarrollo económico local y descentralización en América Latina". Santiago, Chile,

Vázquez Barquero, Antonio (2007). Desarrollo endógeno. Teorías y políticas de desarrollo territorial. Investigaciones Regionales, núm. 11, pp. 183-210 Asociación Española de Ciencia Regional Madrid, España.

Processo Editorial / Editorial Process

Editor Chefe/Editor-in-chief: PhD Thiago D. Pimentel (UFJF).

Recebido em 21 de Novembro de 2017; aceito em 15 de Dezembro de 2017; publicado online 05 de Fevereiro de 2018.

Received on November 21, 2017; accepted on December 15, 2017, published online on February 05, 2018.

Texto original/ Original paper. Sistema de revisão cega por pares / Double blind review system. 